\title{
The Development of Retinopathy in Sucrose-Fed and Streptozotocin-Diabetic Rats
}

\author{
D. Papachristodoulou, H. Heath and S. S. Kang* \\ Department of Biochemical Pathology, University College Hospital Medical School, London, England
}

\begin{abstract}
Summary. Normal and streptozotocin-diabetic rats have been maintained for 6-11 months on completely balanced, reconstituted diets in which the sole source of carbohydrate was either $68 \%$ corn starch or $68 \%$ sucrose. The retinal vascular system was isolated by trypsin digestion and examined histologically for the presence of tortuosity and irregularity of capillary diameter, increased PAS-positive deposits, microaneurysms, loss of pericytes, endothelial proliferation, acellularity and strand formation. None of these pathological changes occurred in normal rats fed a starch-rich diet, but all developed to a similar extent in the sucrose-fed normal rats and the starch-fed diabetic group. The changes were more severe in sucrose-fed diabetic rats after 6 months. In all groups the retinopathy progressed with time. The possibility that a factor common to both the ingestion of a sucroserich diet and streptozotocin diabetes in rats has been considered since, histologically, the retinopathy observed was identical both with respect to severity and rate of development in normoglycaemic, sucrose-fed and hyperglycaemic, starch-fed diabetic rats.
\end{abstract}

Key words: Sucrose-diet, streptozotocin-diabetes, retinopathy, rats, trypsin digests.

Leuenberger and collaborators have reported loss of pericytes and endothelial cells, fusiform microaneurysms, mesodermal bridges and variable thickness of basement membrane in the retinae of streptozotocindiabetic rats after 6-12 months [1-5] and the changes reported were confirmed by the studies of Sosula et al. [6] and Watanabe [7]. Arison's group [8] and von Sallmann and Grimes [9], however, find no retinal lesions resembling human diabetic retinopathy in

\footnotetext{
* Present address: Dept. of Physiologie, Queen Elizabeth College,
} London, England streptozotocin-diabetic rats after 4 and 12 months, respectively. The feeding of a sucrose-rich diet to normal rats for up to 12 months has been reported to produce a nephropathy $[10,11]$ and a retinopathy consisting of varicose loop formation, microaneurysms, irregularity of capillary diameter, strand formation, nonselective dimunution in the number of capillary cells and degeneration of retinal photorecepter cells, that is, changes resembling human diabetic retinopathy $[12,13,14]$. Further experiments on the feeding of sucrose to rats, genetically selected for low glucose tolerance $[15,16]$, have shown an interaction between genetic and environmental factors in the development of angiopathy in diabetes. It seems, then, that the importance of factors not inherent in the idiopathic diabetic state connot be ignored and if such factors could be superimposed on the experimentally induced diabetes they might expose, precipitate or accelerate processes to which the diabetic animal may be predisposed. It was, therefore, decided to study the effect of a high-sucrose diet on the retinae of both normal and diabetic rats in order to determine

a) Whether the ingestion of a high-sucrose diet alone gives rise to a retinopathy in rats similar to human diabetic retinopathy

b) Whether the superimposition of a high-sucrose diet on experimental diabetes exacerbates or accelerates any changes that might be produced in the retina by the diabetic state itself.

\section{Materials and Methods}

\section{Animals}

48 2-month old male Wistar rats (180-250 g) were randomly divided into 4 groups and those from 2 groups were rendered diabetic by the intravenous in- 
Table 1. Body weights, blood glucose levels and degree of retinopathy of the 4 groups of rats at the time of sacrifice

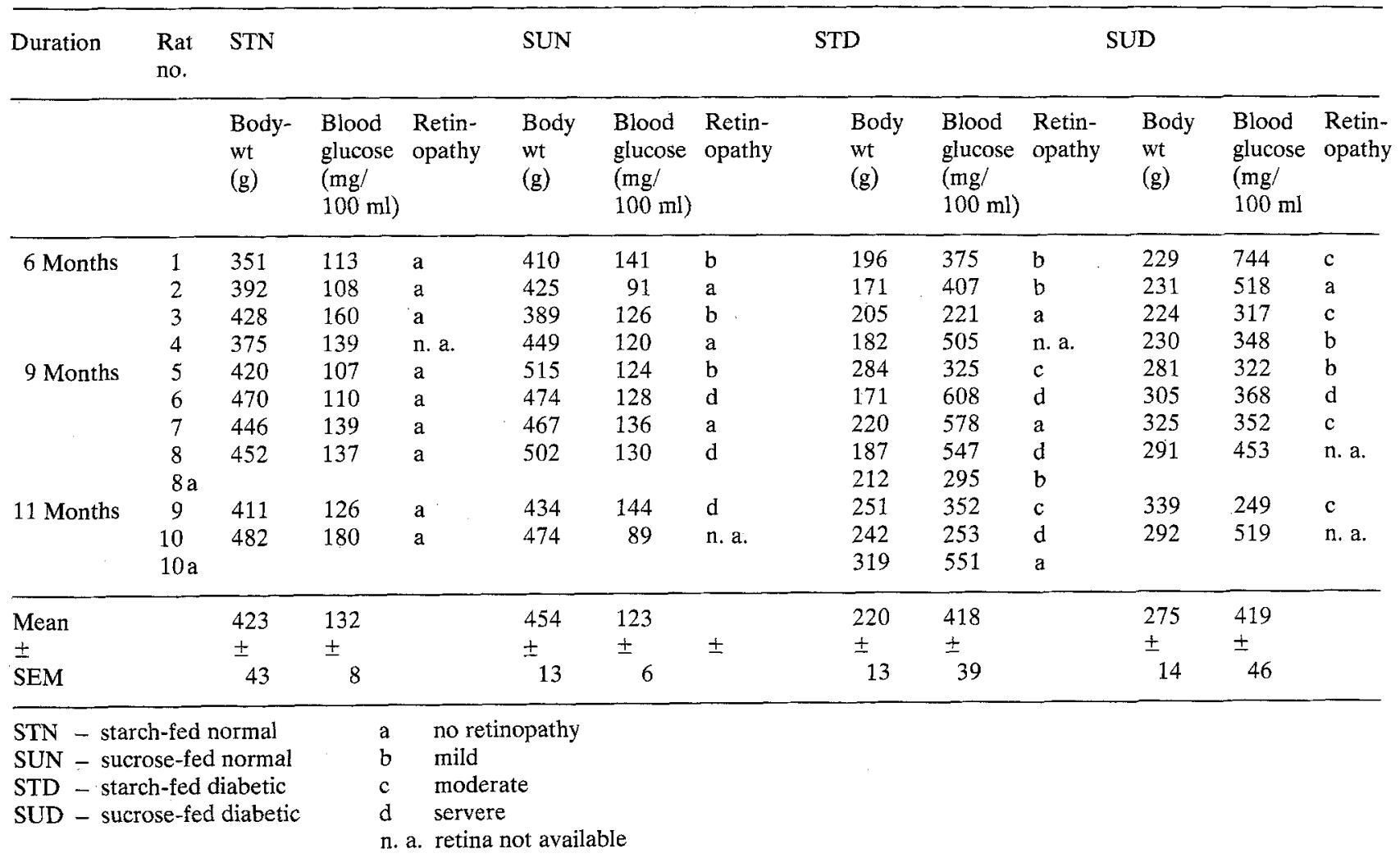

jection of $50 \mathrm{mg}$ streptozotocin $/ \mathrm{kg}$ body weight. Diabetes was established by the occurrence of polyuria, glucosuria and impaired growth. 1 normal and 1 diabetic group were maintained on each of 2 reconstituted diets, in which the sole source of carbohydrate was either corn starch or sucrose $(68 \%$ by weight) [17], for 6 to 11 months. Food and water were administered ad libitum and the rats were periodically weighed and tested for glucosuria with Clinistix.

\section{Preparation of Tissues}

Non-fasted rats were anaesthetised by the intraperitoneal injection of Nembutal $(7.2 \mathrm{mg} / 100 \mathrm{~g}$ body weight) and the eyes were enucleated. One eye was fixed in glutaraldehyde for subsequent electron microscopical examination and the other in neutralised $10 \%$ formol saline solution. Blood was collected by cardiac puncture for glucose determinations. The eyes were kept in formol saline for at least two weeks. The retinae were dissected, washed in distilled water overnight and digested in trypsin by the method of Kuwabara and Cogan [18]. The isolated vascular systems were stained with PAS/haematoxylin. The retinal preparations were examined for pathological changes in ignorance of the group to which they belonged.

\section{Results}

\section{General Observations}

Although the diabetic rats were in a poor condition, mortality was low and not higher than in the normal animals. The diabetic rats ate greater quantities of both starch- and sucrose-rich diets than the nondiabetic rats, but there was no preferential consumption of either diet within the normal or diabetic groups. Cataracts started developing in diabetic rats after 3 months and by 6 months were present in all the diabetic animals. Proliferation of corneal vessels became evident around the 5 th month and was present in all the diabetic rats by the 9 th month. No cataracts or corneal vascularization could be detected in either nondiabetic group.

The body weights of individual rats in each group at the time of sacrifice are shown in Table 1. As rats were randomly divided among the 4 groups initially, the mean starting body weights were similar. Growth was impaired in the diabetic as compared to the normal rats. The mean body weights of the starch-fed normal rats (STN) and the sucrose-fed normal rats (SUN) were not significantly different $(423 \pm 13 \mathrm{~g}$ for STN; $454 \pm 13 \mathrm{~g}$ for SUN), but the mean weights of the sucrose-fed diabetic rats (SUD) were greater than 
Table 2. Features present in the 4 groups of trypsin-digested retinae after 6-11 months of sucrose feeding and/or diabetes

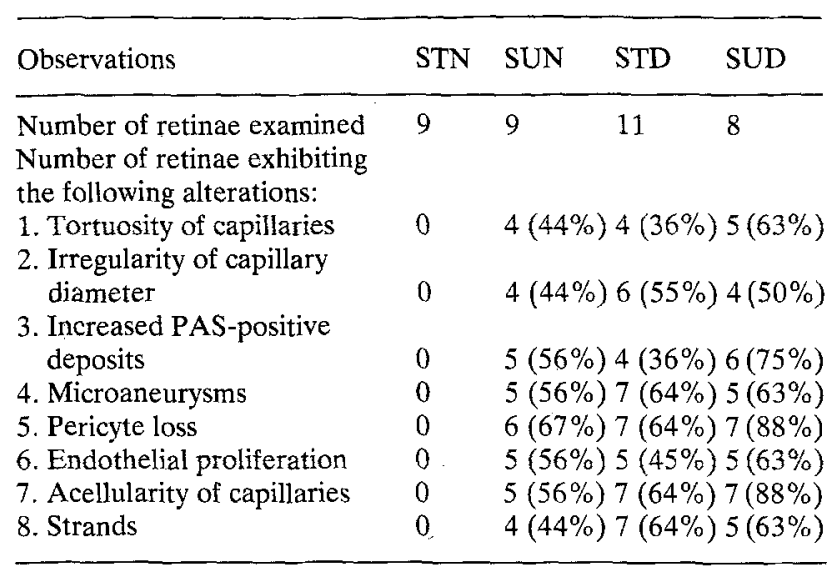

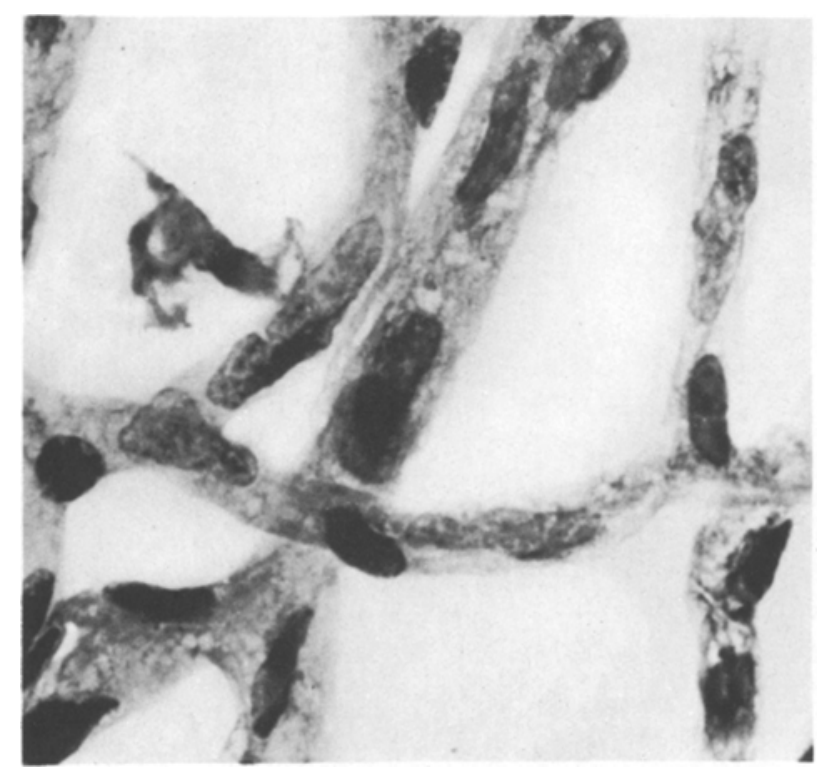

Fig. 2. Capillaries from the retinal vascular system of a normal rat fed a starch-rich diet for 9 months showing normal proportions of pericytes and endothelial cells. $(\times 750)$

those of the starch-fed diabetic rats (STD) $(275 \pm 14 \mathrm{~g}$ for SUD compared with $220 \pm 13 \mathrm{~g}$ for STD; $\mathrm{p}<$ $0.01)$. Table 1 also shows the blood glucose levels for the 4 groups of rats at the time of sacrifice, that is, at 6 , 9 and 11 months respectively. It can be seen that taking the groups as a whole the means of the 2 normal groups are remarkably similar (STN: $132 \pm 9 \mathrm{mg} / 100$ $\mathrm{ml}$; SUN: $123 \pm 6 \mathrm{mg} / 100 \mathrm{ml}$ ) and the same is true of the 2 diabetic groups (STD: $418 \pm 39 \mathrm{mg} / 100 \mathrm{ml}$; SUD: $419 \pm 46 \mathrm{mg} / 100 \mathrm{ml}$ ). There was, however, a

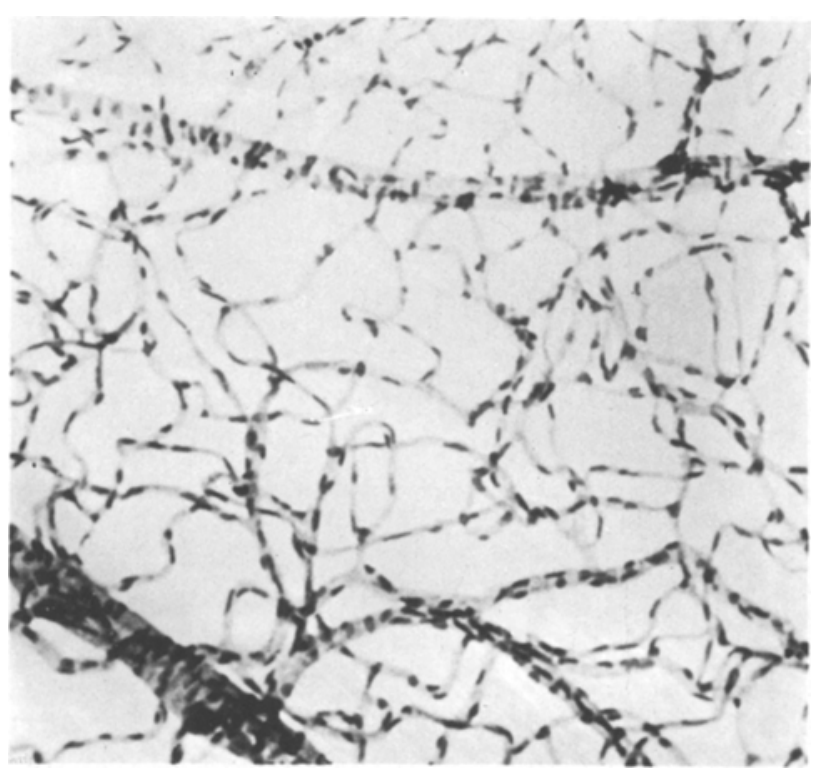

Fig. 1. Part of the retinal vascular system from a normal rat fed a starch-rich diet for 9 months. $(\times 145)$

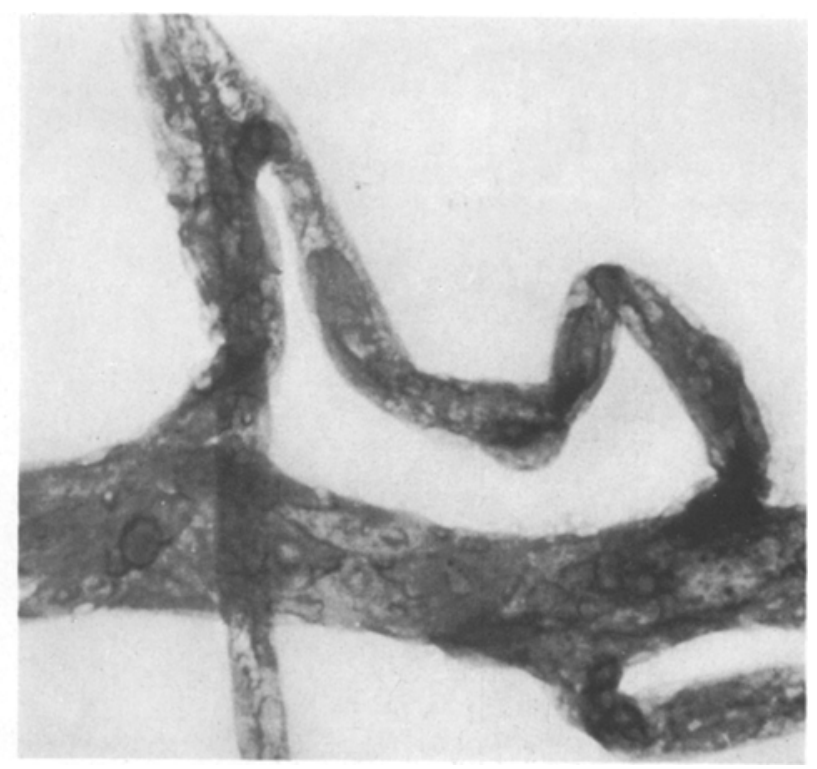

Fig. 3. Outpouching on an acellular capillary from the retinal vascular system of a normal rat fed a sucrose-rich diet for 6 months. $(\times 750)$

great spread in the blood glucose levels among the 2 diabetic groups, with values ranging between $221-600 \mathrm{mg} / 100 \mathrm{ml}$ for the starch-fed diabetic rats and $249-744 \mathrm{mg} / 100 \mathrm{ml}$ in the sucrose-fed diabetic rats.

\section{Histological Results}

All the retinae were examined after trypsin digestion under the light microscope particularly for the occur- 


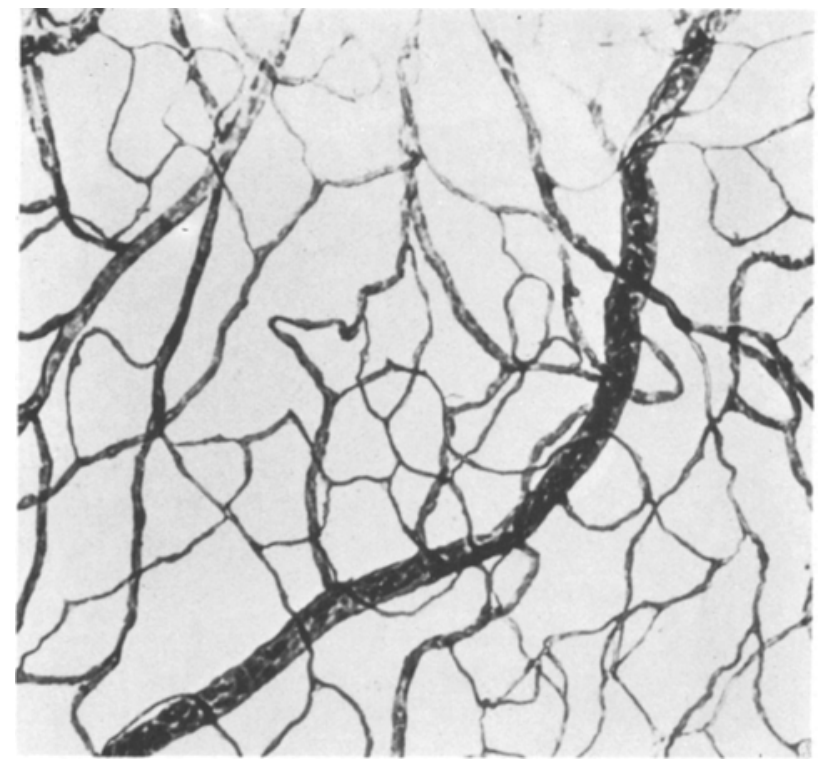

Fig. 4. Part of the retinal vascular system from a normal rat fed a sucrose-rich diet for 11 months showing extensive degeneration of the capillary bed. $(\times 135)$

rence of tortuosity of capillaries, irregularity of capillary diameter, increased PAS-positive deposits, microaneurysms, loss of pericytes, endothelial proliferation, acellularity of capillaries and presence of strands. The distribution of such changes among the various retinae is quantitatively presented in Table 2 . Table 1 shows the degree of retinopathy in individual retinae.

Starch-Fed Normal Rats. Examination of the retinae after 6 months of feeding showed a uniform distribution of PAS staining in arteries, veins and their connecting capillary plexus (Fig. 1). The capillaries were denser near the macula than the periphery and had regular diameters. The nuclei of the 2 types of cells present in retinal vessels were clearly defined. Large, pale-staining ellipsoid nuclei of endothelial cells and darker-staining round nuclei of pericytes could be seen in the normal ratio of 1:1 (Fig. 2). A few intercapillary bridges could be seen connecting the capillaries. These characteristics were retained after 9 and 11 months, with a slight increase in the number of intercapillary bridges, which is a result of the normal process of ageing. No changes that may be taken as pathological could be detected in the retina of starchfed normal rats at any time.

Sucrose-Fed Normal Rats. The feeding of a high sucrose diet to normal rats gave rise to a number of alterations in the appearance of the retinal vasculature. After 6 months of feeding these changes consisted of PAS-positive deposits in some arterioles, tortuosity and irregularity of capillary diameter with localised constrictions and dilatations (Fig. 3), fusiform microaneurysms frequently associated with 'bends' of the capillary and dispersed throughout the vascular bed, selective loss of pericytes and endothelial proliferation in some capillaries and loss of both types of cells in others, often accompanied by degeneration of the capillaries and reduction to strands. Examination after 9-11 months of feeding revealed a marked deterioration in the state of the retina compared to that at 6 months. Tortuosity and irregularity of capillary diameter were more pronounced and pericyte loss and endothelial proliferation affected larger areas of the retina. More microaneurysms could be seen in each affected retina, present usually, but not always, in areas of capillaries devoid of pericytes, although the individual capillaries showing the aneurysmal outpouchings had a reduced number of pericytes. The most striking alteration, however, evident after 9-11 months, was the marked acellularity of many capillaries and their reduction to strands. These acellular changes were not focal in nature, but spread over large areas and they involved groups of capillaries along their entire length. In some areas, especially near the periphery, large areas were replaced by these strands and no functional capillaries could be recognised (Fig. 4). No microaneurysms could be detected on these occluded, degenerating vessels, which seemed to have a predilection for the deeper layers of the vascular network, that towards the choroid. There did not seem to be any specific association with the arterial side of the circulation.

Starch-Fed Diabetic Rats. The changes observed in the starch-fed diabetic rats after six months of diabetes were of similar nature to those seen in sucrose-fed normal rats killed at the same time. No lesion was peculiar to either group and the frequency and extent to which the alterations occurred was very similar in both groups. Moreover, the changes seemed to progress at the same rate. Examination after 9-11 months showed a marked deterioration in the state of the retina of starch-fed diabetic rats, similar to that exhibited by the sucrose-fed rat retinae. Acellular changes, which were so marked in the sucrose-fed normal rat retinae, were equally widespread (Fig. 5) and severe in the starch-fed diabetic rat retinae, and PAS-positive deposits, tortuosity and irregularity of capillaries, fusiform microaneurysms (Fig. 6), diminution in the number of pericytes, endothelial proliferation and acellularity showed a distribution and severity similar to that of the sucrose-fed normal group.

Sucrose-Fed Diabetic Rats. The changes observed in the retinae of the sucrose-fed diabetic rats killed at 6 


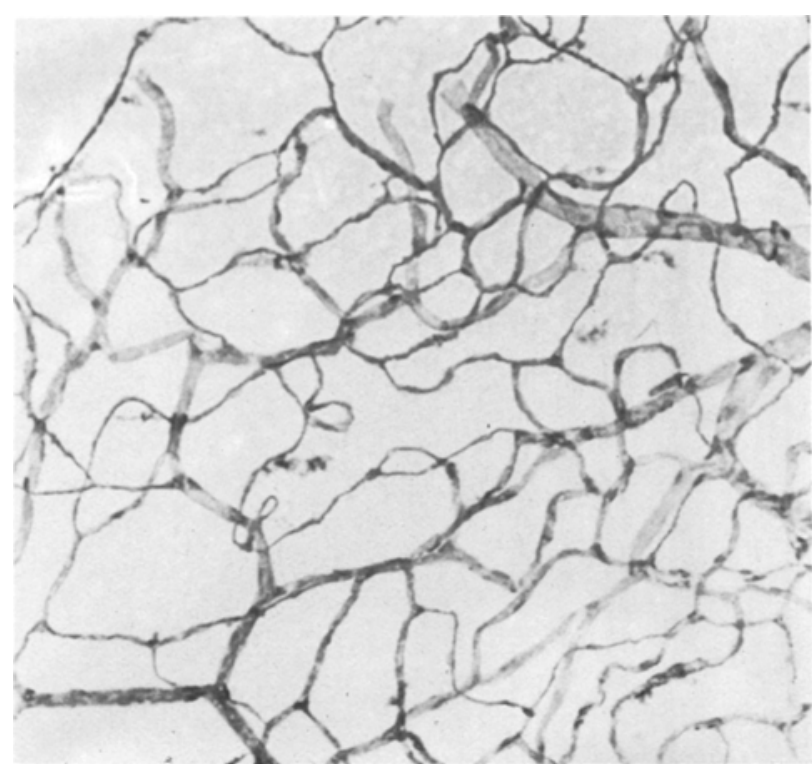

Fig. 5. Part of the retinal vascular system from a streptozotocindiabetic rat fed a starch-rich diet for 9 months showing marked acellularity and degeneration. ( $\times 135)$

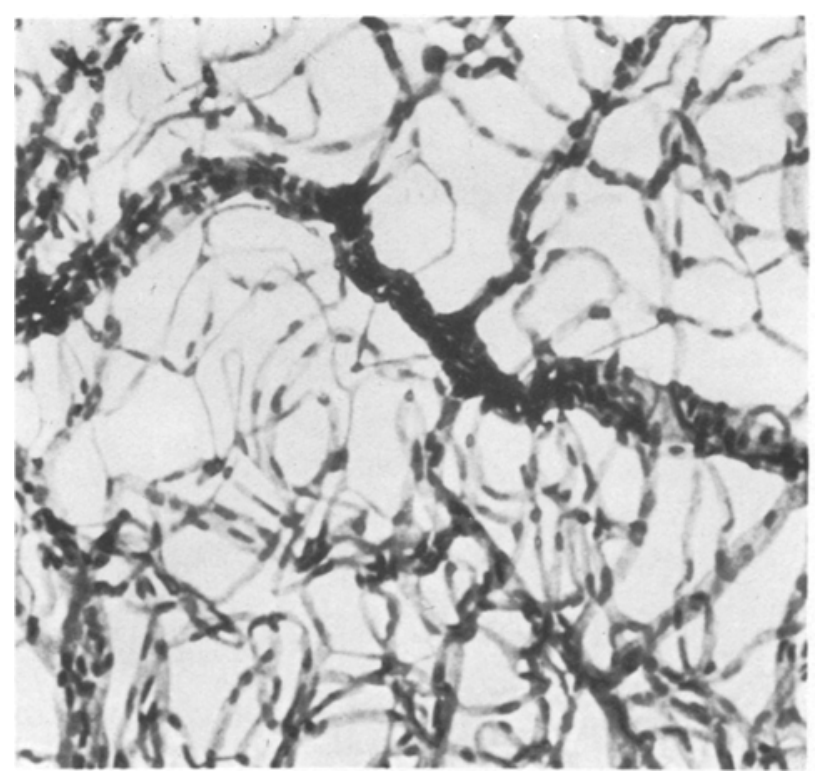

Fig. 7. Part of the retinal vascular system from a streptozotocindiabetic rat fed a sucrose-rich diet for 6 months showing heavy PAS-positive deposits on the arteriole and the presence of capillary strands. $(\times 180)$

months were more pronounced than in sucrose-fed normal or starch-fed diabetic rat retinae at this stage. There were more PAS-positive deposits, mainly on arterioles (Fig. 7), and capillaries were more irregular. Microaneurysms and diminution in the number of pericytes were more frequently encountered, although no saccular microaneurysms could be de-

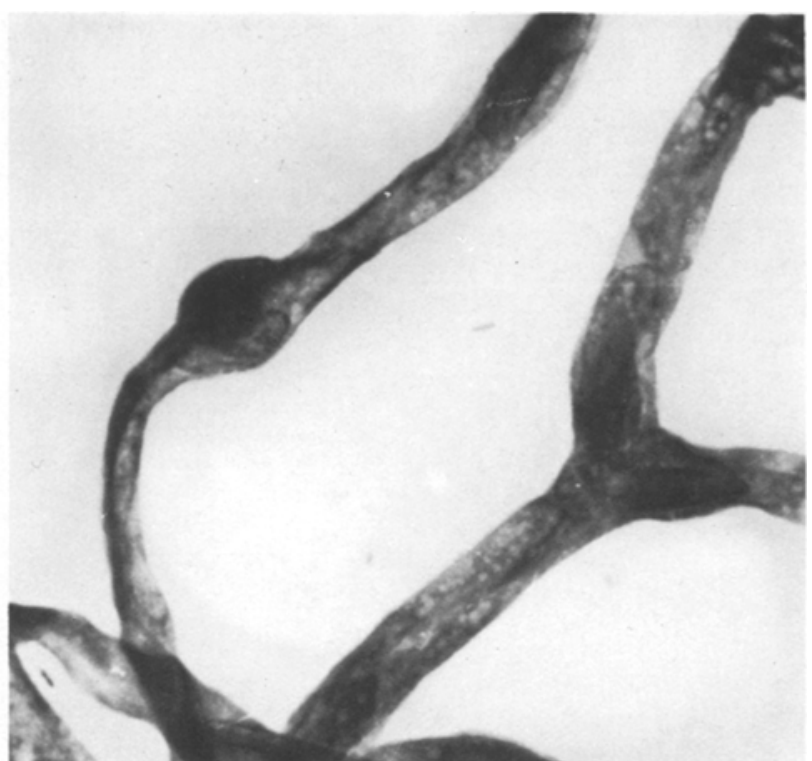

Fig. 6. Capillaries from the retinal vascular system of a streptozotocin-diabetic rat fed a starch-rich diet for 9 months showing a microaneurysm and absence of pericytes. $(\times 1000)$

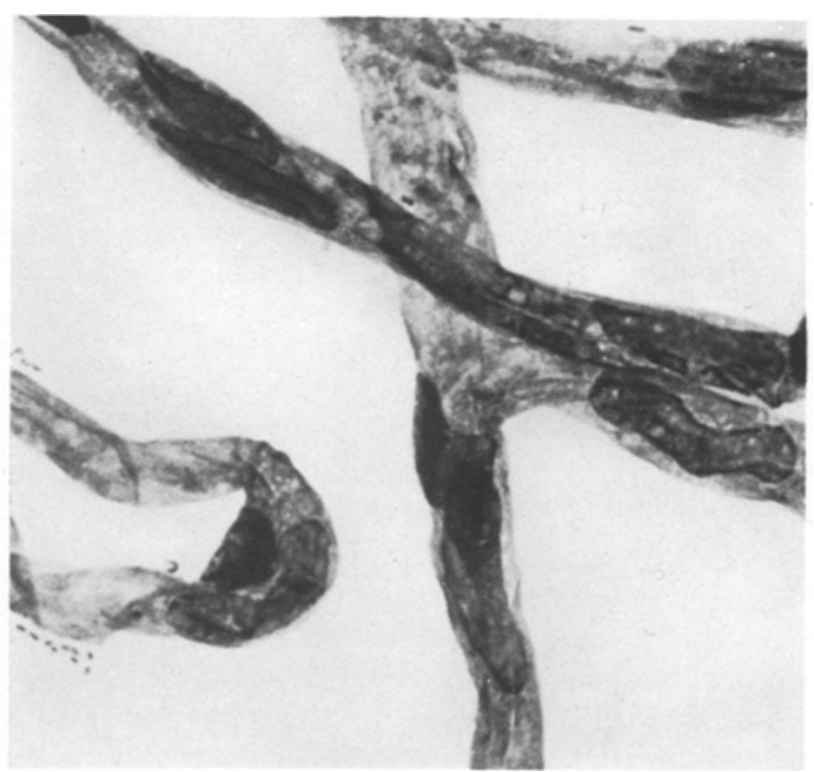

Fig. 8. Capillaries from the retinal vascular system of a streptozotocin-diabetic rat fed on a sucrose-rich diet for 9 months showing a fusiform microaneurysm and selective loss of pericytes. $(\times 1000)$

tected. Acellular capillaries, reduced to strands of basement membrane, were more frequent in any one retina and occupied greater areas of the affected retinae in the sucrose-fed diabetic rats than in the other experimental groups. These strands were intermingled with apparently completely normal capillaries and appeared to be present mainly in the deeper layer of 
the retina, that is, the part nearer the choroid. The state of the retinae markedly deteriorated after 9-11 months (Fig. 8). The pathological changes at this later stage were, however, similar to those exhibited by the sucrose-fed normal and starch-fed diabetic rats, whereas, at 6 months, the retinopathy of the sucrosefed diabetic rats seemed to have progressed faster than the other 2 experimental groups.

\section{Discussion}

As can be seen from the results the feeding of sucrose as the sole source of carbohydrate in an otherwise completely balanced diet resulted in the development of a retinal microangiopathy. Also, as far as could be inferred from the study of the trypsin-digested and PAS/haematoxylin-stained preparations, the retinal lesions produced by the feeding of a high-sucrose diet to normal rats were indistinguishable in frequency, nature and severity from the lesions observed in rats rendered diabetic with streptozotocin and fed on a control high-starch diet for the same length of time. Superimposition of sucrose feeding onto streptozotocin-induced diabetes led to an acceleration in the appearance of the retinal alterations but, although the state of the retina in the sucrose-fed diabetic rats was worse after 6 months than either of the other two experimental groups, the retinopathy of all three groups progressed to a similar degree by the ninth month.

This observed cessation of an additive or, at least, reinforcing effect of the two combined treatments after the initial acceleration of the syndrome may be due to a number of factors, as many variables are involved in experiments of this kind. It must be borne in mind that the retinae were not totally unselected, in that the rats which died spontaneously during the course of the experiment could not be included and, furthermore, the diabetic rats were not randomly selected for sacrifice at 6 months. Those in the poorest state of health were killed first, so comparison at 9 and 11 months, as far as the diabetic rats are concerned, was essentially between the healthier survivors. Also, as two sucrosefed diabetic rats which died spontaneously did so around the sixth month and were in a poor state of health before death, it may be that the remaining sucrose-fed diabetic rats were by far 'healthier' than their starch-fed counterparts, all of which survived.

Thinking along these lines, however, may presume a relation between the state of health in the diabetic rats (which depends on the severity of diabetes) and the expected deterioration in the retina and such a relation is not established. The sucrose-fed diabetic rats were healthier than the starch-fed diabetic rats only as far as growth is concerned, presumably due to their ability to utilize the fructose moiety of their carbohydrate, whose metabolism, unlike that of glucose, is insulin independent. The blood glucose levels of the two diabetic groups were, in fact, remarkably similar $(418 \pm 39 \mathrm{mg} / 100 \mathrm{ml}$ for STD; $419 \pm 46$ $\mathrm{mg} / 100 \mathrm{ml}$ for SUD), so the sucrose-fed diabetic rats, although heavier, were not less severely diabetic.

As retinopathy, however, was not present in all the diabetic rats consideration of mean values of blood glucose only may not be meaningful and the rats have to be considered individually. Retinopathy as a condition cannot be quantified in absolute terms due to the number of abnormalities considered and the varying degree to which different ones were observed in each retina; at the most it can be arbitrarily designated as mild, moderate or severe. Table 1 shows how the presence, absence or degree of retinopathy is related to terminal blood glucose. No direct correlation could be established between the two in either diabetic group. It is apparent from the results that the crucial factor in the development of the retinal vascular abnormalities is not so much the degree of hyperglycaemia as the duration of diabetes. This inference is consonant with the results of the sucrose-fed normal rats. The first important observation in this group of animals was that a retinopathy was produced by the ingestion of high levels of a common human dietary constituent, sucrose, without any elevation in blood glucose levels compared with the controls. The second important observation was that the retinopathy of the sucrose-fed rats was indistinguishable from that of the diabetic rats, with no features peculiar to one group alone. This similarity of the lesions and the suggestion from the results that hyperglycaemia per se may not be crucial leads to a supposition that a common factor may be involved at some stage, but histology alone is incapable of elucidating this point. The concept of a common denominator would be in agreement with the fact that superimposition of sucrose feeding onto streptozotocin diabetes merely accelerates the appearance of retinopathy, but does not exacerbate it once established. At this stage it cannot be concluded what this common factor may be. It seems that it is not glucose. Diabetes, however, is only characterised by hyperglycaemia; it is not simply equivalent to it. It may be that one of the other manifestations of the diabetic state relates to the results of the ingestion of high levels of sucrose and its identification may provide the missing link.

The retinae of these rats were not available for biochemical analysis; therefore, another series of animals was maintained for 20 days on the same regimens and the retinae analysed for adenine nucleotides, since it has been reported that alloxan diabetes [19] 
and infusions of fructose [20] cause a decrease in ATP and an increase in ADP and AMP content of the liver. No changes, however, occurred in the ATP, ADP, AMP and inorganic phosphate content of the retinae from either sucrose-fed or diabetic rats [21]. It would appear, therefore, that aberration in adenine nucleotide metabolism is not responsible for the observed retinopathy. The fructose and sorbitol content of the retinae have also been analysed in a similar series of rats after 15 days on reconstituted diets. The diabetic state significantly increased the levels of sorbitol and fructose in the retina, but sucrose feeding to normal rats did not cause such an elevation [22]. The retinopathic changes observed were probably, therefore, not due to an increased sorbitol content in the retina. The flux of glucose through the sorbitol pathway has not been determined and, therefore, the rate of fructose metabolism in the retina cannot be assessed. This may have a bearing on the development of a retinopathy, since we have observed a significant increase in lactic acid content in the retinae from sucrose-fed normal and starch- or sucrose-fed diabetic rats when compared to the concentration in the starch-fed normal animals [17]. It was, therefore, in the animals which were prone to an increase in retinal lactic acid that retinopathy developed. Lactic acid has been implicated by Keen and Chlouverakis [23] and Imre [24] in the development of retinopathy, but since the observed retinopathy in our animals could lead to an anoxic state, the time sequence of lactate accumulation/vascular changes cannot be firmly established.

In the human diabetic condition retinopathy proceeds to a much more advanced stage, especially as regards microaneurysms and new vessel formation, than that observed in our experimental animals, but this may be due either to the shorter period of the inception of retinopathy, which could explain the absence of neovascularization, or to some intrinsic difference between rat and human retinal metabolism. Even though new retinal vessel formation and development of numerous microaneurysms did not take place, the other pathological changes are identical with those occurring in the human diabetic condition. It would appear, therefore, that both sucrose retinopathy and streptozotocin-diabetic retinopathy in rats offer suitable models for studying the aetiology of human diabetic retinopathy, since the initial stimulus may in all cases be the same, though some property of the rat retinal capillary circulation may preclude these vessels from deteriorating in the same manner as human retinal capillaries.

Acknowledgements. The authors are grateful for the financial support received from the British Founda- tion for Research into the Prevention of Blindness and the Clothworkers' Company and for the gift of streptozotocin from Upjohn Ltd. We wish to thank Professor N. Ashton for his valuable help and advice and Miss J. R. Hodsdon for her co-operation and assistance.

\section{References}

1. Leuenberger, P., Cameron, D., Stauffacher, W., Renold, A. E., Babel, J.: Ocular lesions in rats rendered chronically diabetic with streptozotocin. Ophthal. Res. 2, 189-204 (1971)

2. Cameron, D. P., Leuenberger, P., Amberdt, M., Mira, F., Orci, L., Stauffacher, W.: Microvascular lesions including retinal aneurysms in chronic experimental diabetes (streptozotocin). Europ. J. clin. Invest. 1, 365 (1971)

3. Cameron, D. P., Amherdt, M., Leuenberger, P., Orci, L., Stauffacher, W.: Microvascular alterations in chronically streptozotocin-diabetic rats. Advanc. Metabolic Disorders 2 (Suppl.), 257-269 (1973)

4. Leuenberger, P. M., Beauchemin, M. L., Babel, J.: Experimental diabetic retinopathy. Arch. Ophtal. (Paris) 34, 289-302 (1974)

5. Babel, J., Leuenberger, P.: A long term study on the ocular lesions in streptozotocin diabetic rats. Albrecht v. Graefes Arch. Ophthal. 189, 191-209 (1974)

6. Sosula, L., Beaumont, P., Hollows, F., Jonson, K.: Dilatation and endothelial proliferation of retinal capillaries in streptozotocin-diabetic rats: Quantitative electron microscopy. Invest. Ophthal. 11, 926-935 (1972)

7. Watanabe, R.: Histological studies of the retinae of rats with streptozotocin-induced diabetes. Acta Soc. ophthal. jap. 77, 605-614 (1973)

8. Arison, R. N., Glaccio, E. I., Glitzer, M. S., Cassaro, A. B., Pruss, M. P.: Light and electron microscopy of lesions in rats rendered diabetic with streptozotocin. Diabetes 16, 51-56 (1967)

9. von Sallmann, L., Grimes, P.: Histopathologic changes in the eyes and kidneys of rats with long term streptozotocin diabetes. In: Contemporary ophthalmology honoring Sir Stewart DukeElder (ed. J. Bellows), p. 502. Baltimore: Wilkin and Wilkin Co. 1972

10. Rosemann, E., Teitelbaum, A., Cohen, A. M.: Nephropathy in sucrose-fed rats. Electron and light microscopic studies. Diabetes 20, 803-810 (1971)

11. Cohen, A. M., Rosenmann, N.: Diffuse capillary glomerulosclerosis in the sucrose-fed rat. Diabetologia 7, 25-28 (1971)

12. Cohen, A. M., Michaelson, I. C., Yanko, L.: Retinopathy in rats with disturbed carbohydrate metabolism following a high sucrose diet. Amer. J. Ophthal. 73, 863-869 (1972)

13. Yanko, L., Michaelson, I. C., Cohen, A. M.: Retinopathy in rats with disturbed carbohydrate metabolism following a high sucrose diet. Amer. J. Ophthal. 73, 870-875 (1972)

14. Rosenmann, E., Yanko, L., Cohen, A. M.: Comparative study of the pathology of sucrose-induced diabetes in the rat and adult-onset diabetes in man. Israel J. med. Sci. 11, 753-761 (1975)

15. Cohen, A. M., Teitelbaum, A., Saliternik, R.: Genetics and diet as factors in development of diabetes mellitus. Metabolism 21, 235-252 (1972)

16. Cohen, A. M., Teitelbaum, A., Briller, S., Rosenmann, E., Yanko, L., Shafrir, E.: Role of diet and genetics in the development of angiopathy in diabetes. Horm. Metab. Res. 6 (Suppl.), $117-123$ (1974) 
17. Heath, H., Kang, S. S., Philippou, D.: Glucose, glucose-6-phosphate, lactate and pyruvate content of the retina, blood and liver of streptozotocin-diabetic rats fed sucrose- or starch-rich diets. Diabetologia 11, 57-62 (1975)

18. Kuwabara, T., Cogan, D. G.: Studies of retinal vascular patterns. I. Normal architecture. Arch. Ophthal. 64, 904-911 (1960)

19. Blaylock, B. A., Rothrock, E., Sacks, J.: Alloxan diabetes, insulin and adenosine phosphates in the liver. Amer. J. Physiol. 198, 1063-1065 (1960)

20. Woods, H. F., Alberti, K. G. M. M.: Dangers of intravenous fructose. Lancet 1972 II, 1354-1357

21. Papachristodoulou, D., Heath, H.: Effect of sucrose feeding and diabetes on adenine nucleotide and phosphate content of rat retina, liver and blood. IRCS Med. Sci. 3, 402 (1975)

22. Heath, H., Hamlett, Y. C.: The sorbitol pathway: Effect of streptozotocin-induced diabetes and the feeding of a sucrose- rich diet on glucose, sorbitol and fructose in the retina, blood and liver of rats. Diabetologia 12, 43-46 (1976)

23. Keen, H., Chlouverakis, C.: Metabolic factors in diabetic retinopathy. In: Biochemistry of the retina (ed. C. N. Graymore), p. 134. London and New York: Academic Press 1965

24. Imre, G.: Studies on the mechanism of retinal neovascularization: Role of lactic acid. Brit. J. Ophthal. 48, 75-82 (1964)

Received: February 20, 1976, and in revised form: May 28, 1976

Dr. H. Heath

Department of Biochemical Pathology

University College Hospital Medical School

University Street

London WC1E 6JJ

England 